\title{
Hypervelocity Impact Performance of 3D Printed Aluminum Panels
}

\author{
B. A. Davis ${ }^{1}$, R. A. Hagen ${ }^{2}$, R. J. McCandless ${ }^{1}$, E. L. Christiansen ${ }^{3}$, and D. M. Lear ${ }^{3}$ \\ ${ }^{1}$ Jacobs, NASA Johnson Space Center, Mail Code XI, Houston, TX 77058, USA, \\ ${ }^{2}$ NASA Johnson Space Center, Mail Code ES, Houston, TX 77058, USA \\ ${ }^{3}$ NASA Johnson Space Center, Mail Code XI, Houston, TX 77058, USA
}

\begin{abstract}
With the continued development of additive manufacturing methods, control over the shape of ligaments, cell regularity, and macroscopic shape can all be easily tuned. This capability allows for tailoring of component architecture and promotes potential mass savings in a space vehicle structure. Additionally, it allows one the flexibility of combining structural elements such as MMOD protection and vehicle stiffness for launch loads for an overall mass reduction. At NASA JSC this technology is being explored in many different ways with the goal being a multifunctional structural component. For this study, four different types of aluminum panels have been 3D printed for testing, three being of a body centric cubic (BCC) lattice structure core and one being kelvin cell structure core. All samples have a $5.33 \mathrm{~cm}\left(0.05^{\prime \prime}\right)$ nominally thick aluminum face sheet printed on the front and back side of each panel, with all core materials having a $5.08 \mathrm{~cm}(2.0$ ") nominal thickness (see Table 1 for test sample summary and Figures $1-2$ for sample illustrations). These tests will evaluate the performance of 3D printed aluminum panels under hypervelocity impact (HVI) conditions.
\end{abstract}

\begin{tabular}{|c|c|c|c|c|c|c|c|}
\hline \multicolumn{8}{|c|}{ Table 1 - Test Sample Summary } \\
\hline $\begin{array}{c}\text { Sample } \\
\text { serial number }\end{array}$ & $\begin{array}{l}\text { Panel } \\
\text { core type }\end{array}$ & $\begin{array}{c}\text { Core } \\
\text { dimensional } \\
\text { information } \\
(\mathrm{cm})\end{array}$ & $\begin{array}{c}\text { Sample } \\
\text { pre-test } \\
\text { mass } \\
(\mathrm{g})\end{array}$ & $\begin{array}{c}\text { Sample } \\
\text { post-test } \\
\text { mass } \\
\text { (g) }\end{array}$ & $\begin{array}{c}\text { Overall sample nominal } \\
\text { dimensions } \\
(\mathrm{cm})\end{array}$ & $\begin{array}{c}\text { Mass per } \\
\text { unit area } \\
\left(\mathrm{g} / \mathrm{cm}^{2}\right)\end{array}$ & $\begin{array}{l}\text { Overall } \\
\text { density } \\
\left(\mathrm{g} / \mathrm{cm}^{3}\right)\end{array}$ \\
\hline 1 & $\mathrm{BCC}$ & $\begin{array}{c}0.076(0.030 ") \\
\text { nominal lattice diameter }\end{array}$ & 1150.0 & & $\begin{array}{c}15.24 \mathrm{~L} \text { x } 15.24 \mathrm{~W} \text { x } 5.33 \mathrm{H} \\
(6.0 " \text { x 6.0" x 2.1") }\end{array}$ & 4.951 & 0.929 \\
\hline 2 & Kelvin & $\begin{array}{c}0.076(0.030 ") \\
\text { nominal wall thickness }\end{array}$ & 977.0 & & $\begin{array}{c}15.24 \mathrm{~L} \text { x } 15.24 \mathrm{~W} \times 5.33 \mathrm{H} \\
(6.0 " \times \text { 6.0" x 2.1") }\end{array}$ & 4.207 & 0.789 \\
\hline 3 & $\mathrm{BCC}$ & $\begin{array}{c}0.051(0.020 ") \\
\text { nominal lattice diameter }\end{array}$ & 720.9 & & $\begin{array}{c}15.24 \mathrm{~L} \text { x } 15.24 \mathrm{~W} \text { x } 5.33 \mathrm{H} \\
(6.0 " \times 6.0 " \text { x 2.1") }\end{array}$ & 3.104 & 0.582 \\
\hline 4 & $\mathrm{BCC}$ & $\begin{array}{c}0.064(0.025 ") \\
\text { nominal lattice diameter }\end{array}$ & 922.9 & & $\begin{array}{c}15.24 \mathrm{~L} \text { x } 15.24 \mathrm{~W} \text { x } 5.33 \mathrm{H} \\
(6.0 " \times 6.0 " \mathrm{x} 2.1 ")\end{array}$ & 3.974 & 0.746 \\
\hline
\end{tabular}


The hypervelocity impact tests are being conducted at the JSC White Sands Test Facility

(WSTF) Remote Hypervelocity Test Laboratory (RHTL), located in Las Cruces, New Mexico. All tests will be conducted with a $3.4 \mathrm{~mm} \mathrm{Al} 2017-\mathrm{T} 4$ sphere at $6.8 \mathrm{~km} / \mathrm{s}$ impacting at $0^{\circ}$ to surface normal (i.e., impacting with no obliquity). Each sample will be trapped between two metal frames, with gasket material residing between the sample and frame, which will be the shipping and testing configuration for all tests. There will be an Al 2017-T4 witness plate staged $5.08 \mathrm{~cm}$ (2.0") from each sample to capture signature of debris, if the rear face sheet of the sample were to perforate from the HVI test event.

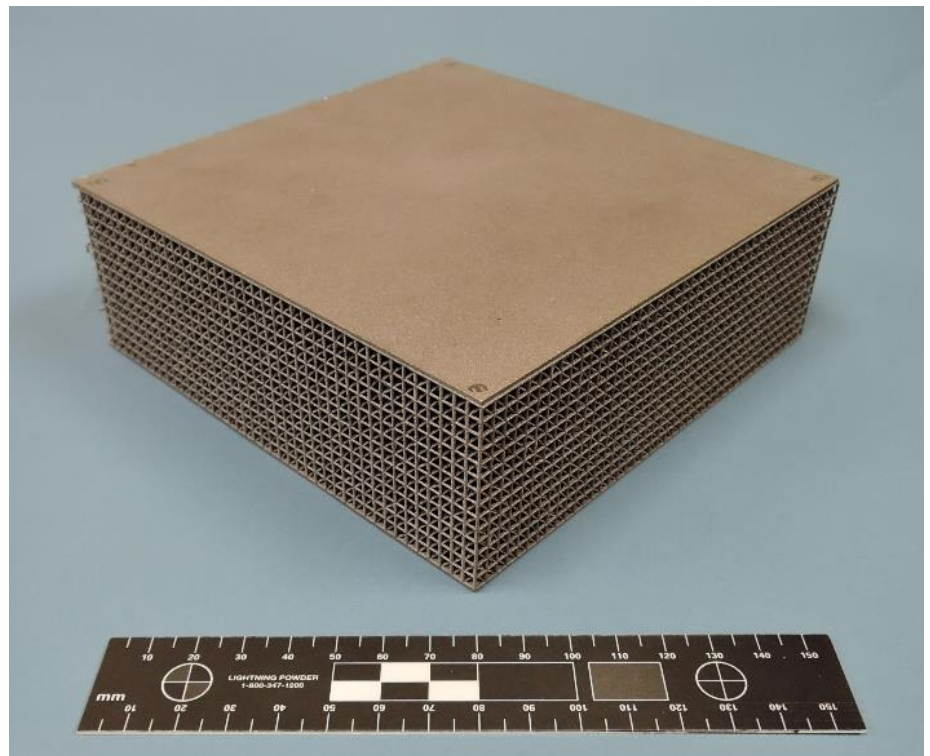

Figure 1 Representative of a BCC lattice structure core panel sample.

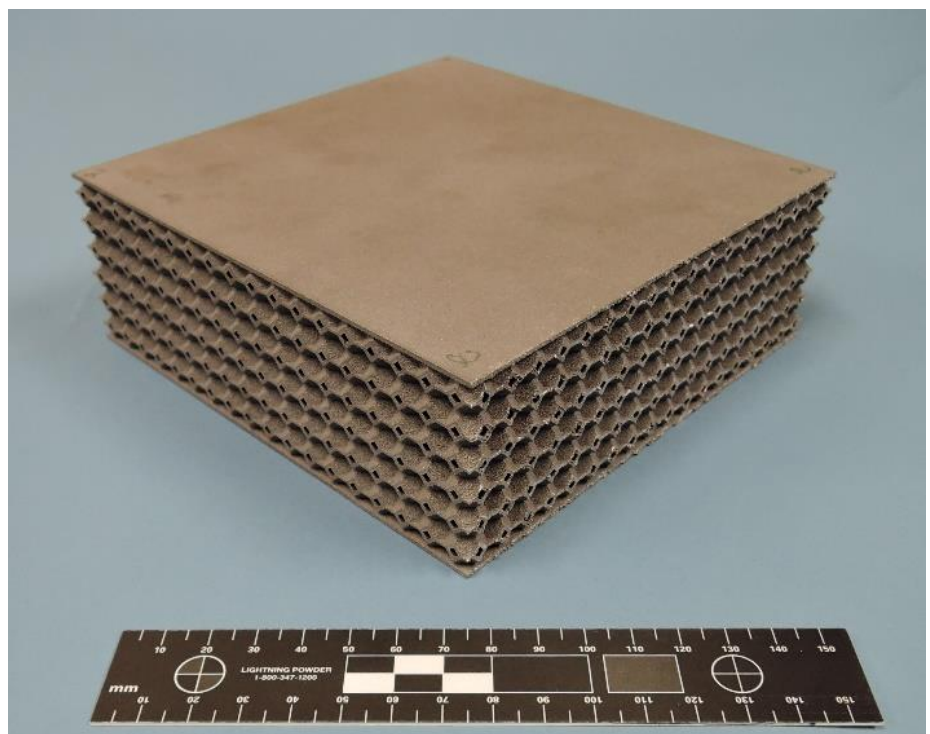

Figure 2 Representative of a Kelvin cell structure core panel sample. 